\title{
Elaboration d'une méthode de test d'interaction digestive entre les composants du régime chez les animaux monogastriques
}

\author{
Sylvie GIGER ${ }^{(*)}$, Béatrice DARCY-VRILLON ${ }^{(*)}$, \\ Nadine MANDRAN ${ }^{(*)}$ et Camille DUBY ${ }^{(* * *)}$ \\ * INRA, Station de Nutrition et Alimentation, I.N.A.-Paris-Grignon, 75231 Paris Cedex 05 \\ ** INRA, Laboratoire de Physiologie de la Nutrition, Centre de Recherches de Jouy-en-Josas, \\ 78350 Jouy-en-Josas \\ *** Chaire de Mathématiques et Informatique, I.N.A.-Paris-Grignon, 75231 Paris Cedex 05
}

\begin{abstract}
Résumé
L'estimation de la valeur nutritive d'un aliment composé est généralement basée sur l'additivité des valeurs nutritives des matières premières entrant dans sa composition. Or, cette approche n'est valable qu'en l'absence d'interaction digestive.

La méthode décrite dans le présent travail permet de tester statistiquement l'existence d'un tel phénomène dans le cas de l'association de deux matières premières, même si le nombre d'aliments expérimentés est supérieur à 2 et quelle que soit la forme de l'interaction.

La démarche proposée comporte deux étapes. Dans un premier temps, une analyse de variance permet de tester statistiquement l'existence éventuelle d'un effet régime et celle d'un effet animal et, s'il y a des répétitions, l'interaction animal-régime. L'absence d'effet régime permet de conclure, dès ce niveau, à une absence d'interaction digestive.

La seconde étape n'est donc entreprise que si l'effet régime est statistiquement significatif. Elle consiste à comparer la somme des écarts des carrés résiduels, obtenus par analyse de variance dans la première étape, avec celle issue d'un modèle d'analyse de covariance qui utilise le taux d'incorporation d'un des composants de la ration comme variable explicative et tient compte de l'effet animal.

Cette méthode est également valable lorsqu'il n'y a pas de répétitions ou lorsque le dispositif est déséquilibré. Elle permet, en outre, de tester les effets liés à l'animal et l'interaction animalaliment et est relativement simple à mettre en ouvre.
\end{abstract}

Mots clés: Interaction digestive, valeur nutritive, composants du régime, porc, monogastriques.

\section{Introduction}

Les animaux monogastriques et, en particulier les porcs, lorsqu'ils sont placés en situation d'élevage intensif, reçoivent en général dans leur ration un mélange de matières premières de nature variée, choisies et incorporées de manière à satisfaire les besoins nutritionnels des animaux. Le mode de formulation du mélange est basé sur le principe de l'additivité des valeurs nutritives des composants : sa valeur nutritive est égale à la somme des valeurs correspondant aux différentes matières premières pondé- 
rée par le taux d'incorporation de chacune dans le mélange considéré. En d'autres termes, cette approche ne prend pas en considération les effets éventuels d'une interaction digestive entre les différents ingrédients de la ration.

Afin de préciser et de cerner les limites de la validité de ce mode de calcul, il est nécessaire de tester sur les animaux les principales matières premières, seules et en les associant selon des pourcentages connus, et de disposer d'une méthode statistique qui puisse tester les éventuelles interactions digestives.

L'objet de la présente étude est de définir une telle méthode dans le cas le plus couramment rencontré par les chercheurs travaillant sur l'alimentation et la nutrition des porcs, c'est-à-dire dans le cas de deux matières premières distribuées seules ou en association au sein d'un mélange.

\section{Matériel et méthodes}

\section{A. Protocole expérimental}

Les données utilisées dans ce travail sont issues d'une expérimentation sur cinq porcs recevant chacun trois régimes différents, respectivement à base de son de blé fin, de coques de soja et d'un mélange constitué de quantités égales de son de blé et de coques de soja, sur la base de la matière sèche (LAPlACE et al., 1988).

Les mesures de digestibilité iléale de la matière sèche et de l'azote correspondent à des mesures journalières ( 3 répétitions/animal/régime), tandis que celles de la matière organique et des acides aminés ont été calculées à partir d'un échantillon moyen par animal et par régime. Parmi les digestibilités des acides aminés, celle de la méthionine a été prise à titre d'exemple.

\section{B. Méthode de test d'une interaction digestive}

1. Première étape: recherche d'une interaction éventuelle animal-régime et d'un effet régime

Cette première étape a pour but de tester sur le coefficient de digestibilité (Y) un éventuel effet du régime ( $\mathrm{Re}$ à $\mathrm{k}$ niveaux), une fois pris en compte l'effet lié à l'animal (An à n niveaux), et, si possible, l'interaction animal-régime (SNEDECOR et Cochran, 1984).

a) Cas de non-répétition des mesures pour un animal donné avec un régime donné

Le modèle statistique correspondant au $\mathrm{i}^{\mathrm{i} \text { ème }}$ porc recevant le $\mathrm{j}^{\mathrm{ième}}$ régime est :

$Y_{i j}=m u+A n_{i}+R_{e}+e_{i j}$

$$
(i=1, n ; j=1, k)
$$

Dans ce modèle, la somme des carrés des écarts (SCE) totale qui a (nk - 1) degrés de liberté (ddl) se décompose en :

- SCE liée à un effet animal à $(n-1)$ ddl ;

- SCE liée à un effet régime à $(\mathrm{k}-1)$ ddl ;

- $\mathrm{SCE}$ résiduelle à $(\mathrm{nk}-\mathrm{n}-\mathrm{k}+1)$ ddl. 
La SCE résiduelle comporte, dans le cas présent, un terme correspondant à une éventuelle interaction régime-porc, mais qu'il n'est pas possible de tester, de façon simple, du fait du manque de répétitions, et donc de degrés de liberté.

Le test des différents effets s'effectue classiquement à l'aide d'un test de Fisher (SNEDECor \& Cochran, 1984).

b) Cas de répétition des mesures pour un animal donné avec un régime donné

Dans le cas où l'on dispose de 1 répétition pour un animal donné avec un régime donné, il est possible d'utiliser le modèle suivant ;

$$
\begin{array}{r}
Y_{i j 1}=m u+A n_{i}+\operatorname{Re}_{j}+(A n * R e)_{i j}+e_{i j 1} \\
(i=1, n ; j=1, k ; 1=1, r)
\end{array}
$$

Dans ce modèle, la SCE totale se décompose en :

- SCE liée à un effet animal à $(n-1)$ ddl ;

- SCE liée à un effet régime à $(\mathrm{k}-1)$ ddl ;

- SCE liée à l'interaction régime-animal à $(\mathrm{n}-1)(\mathrm{k}-1) \mathrm{ddl}$;

- SCE résiduelle à $(\mathrm{nk})(\mathrm{r}-1)$ ddl.

Comme dans le cas précédent, les différents effets sont testés en comparaison à la loi de Fisher.

c) Conclusions à tirer de cette première étape

- Test de l'interaction animal-régime, dans le cas où des répétitions permettent de le tester.

Un effet significatif à ce niveau peut limiter fortement la portée des résultats de l'interprétation, puisque son existence montre que les différents animaux ne réagissent pas de la même façon et que les résultats sont, de ce fait, en partie liés au choix du matériel expérimental utilisé.

- Test de l'effet régime.

En l'absence de signification de ce test au seuil choisi, il est possible de conclure, dès ce niveau, qu'il n'y a pas d'interaction digestive entre les composants de la ration, puisque les régimes ne sont pas statistiquement différents.

- Test de l'effet animal.

Dans le cas où un animal est statistiquement différent des autres, il y a lieu d'examiner attentivement les résultats provenant de cet animal afin de déterminer s'il est ou non aberrant.

\section{Seconde étape: test d'interaction entre les composants du mélange}

Cette seconde étape ne se justifie que dans la mesure où la première étape a mis en évidence un effet régime. Le principe de la méthode utilisée a été décrit, notamment, par Schneider \& Flatt (1975) et Giger \& Sauvant (1983) : à chaque mesure de digestibilité $\mathrm{Y}_{\mathrm{ij}}$, est associée une valeur $\mathrm{x}_{\mathrm{j}}$ correspondant au taux d'incorporation dans le régime d'un des mélanges de base et pour le constituant considéré. Ainsi, dans le cas présent, la valeur de $\mathrm{x}_{\mathrm{j}}$, qui représente le taux d'incorporation des coques de soja, varie de 0 (régime son de blé) à 1 (régime coques de soja) en passant par 0,5 (régime son de blé + coques de soja) dans le cas de la matière sèche ${ }^{(*)}$.

$\left({ }^{*}\right)$ Cette valeur pour le régime mixte (son + coques) varie suivant le constituant considéré. Elle est par exemple de 0,688 pour la cellulose brute $(\mathrm{CB})$, puisque les taux de $\mathrm{CB}$ du son et des coques sont respectivement de $5,75 \% /$ MS et $12,65 \% /$ MS. 
S'il n'y a pas d'interaction entre les composants, la réponse suit le modèle :

$Y_{i j 1}=a+b x_{j}+e_{i j 1}$

où, dans le cas présent, « $a$ » représente la digestibilité du son, et $« a+b$ », celle des coques. Il est possible de transformer ce modèle de régression en modèle d'analyse de covariance en y ajoutant un effet animal :

$$
\mathrm{Y}_{\mathrm{ij} 1}=\mathrm{a}+\mathrm{An}_{\mathrm{i}}+b x_{\mathrm{j}}+\mathrm{e}_{\mathrm{ij} 1}
$$

Le test habituel de la linéarité de la régression consiste à introduire dans cette équation un terme en $\mathrm{x}^{2}$ :

$$
Y_{i j 1}=a+A n_{i}+b x_{j}+c x^{2}+e_{i j 1} \text {, }
$$

comme Giger \& Sauvant (1983) l'ont proposé. Cependant, cette méthode n'est valable que dans le cas où $x$ prend plus de trois valeurs distinctes (ShefFe, 1959 ; SNedecor \& Cochran, 1984), ce qui est le cas dans un certain nombre d'expériences sur ruminants. Dans le cas présent, où il n'y a que 3 valeurs distinctes pour $x$, nous proposons un test basé sur la comparaison des sommes des carrés résiduelles obtenues par analyse de variance avec effet régime et animal (étape 1) et par régression (ou de variancecovariance) avec un terme en $\mathrm{x}$ (étape 2 ), et récapitulé dans le tableau 1 .

\section{TABLEaU 1}

Test de linéarité de la régression.

\begin{tabular}{|c|c|c|c|}
\hline & \multirow{3}{*}{ SCE } & \multicolumn{2}{|c|}{ Nombre de ddl } \\
\hline & & sans & avec \\
\hline & & \multicolumn{2}{|c|}{ répétition } \\
\hline $\begin{array}{l}\text { SCE résiduelle de l'analyse de co- } \\
\text { variance } \ldots \ldots \ldots \ldots \ldots \ldots \\
\text { SCE résiduelle de l'analyse de va- } \\
\text { riance } \ldots \ldots \ldots \ldots \ldots \ldots \ldots \\
\text { Réduction de la } \mathrm{SCE} \ldots \ldots \ldots \ldots\end{array}$ & $\begin{array}{l}\mathrm{SCE}_{\mathrm{vc}} \\
\mathrm{SCE}_{\mathrm{v}} \\
\mathrm{SCE}_{\mathrm{vc}}-\mathrm{SCE}_{\mathrm{v}}\end{array}$ & $\begin{array}{l}\mathbf{n k}-\mathbf{n}-1 \\
\mathrm{nk}-\mathrm{n}-\mathrm{k}+1 \\
\mathrm{k}-2\end{array}$ & $\begin{array}{l}n k r-n-1 \\
n k(r-1) \\
n k-n-1\end{array}$ \\
\hline
\end{tabular}

Test of the linearity of the regression.

Le carré moyen de la réduction de la SCE est ensuite comparé au carré moyen de la résiduelle de l'analyse de variance selon une loi de Fisher à $(k-2, n k-n-k+1) d d l$, s'il n'y a pas de répétition, et à $(\mathrm{nk}-\mathrm{n}-1, \mathrm{nk}(\mathrm{r}-1)) \mathrm{ddl}$, s'il y en a.

Ce dernier test permet de montrer s'il y a une interaction digestive puisqu'il teste une éventuelle non-linéarité de forme quelconque de la régression.

\section{Résultats}

Ce chapitre a pour objet de montrer les différents cas de figures précédemment décrits. 


\section{A. Cas de l'azote}

Les résultats de l'analyse de variance concernant l'azote sont récapitulés dans le tableau 2. Ils montrent qu'il n'est pas possible de mettre statistiquement en évidence, avec ces données, des effets liés au régime ou à l'animal, et qu'il n'y a pas d'interaction régime-animal.

\section{Tableau 2}

Analyse de variance sur la digestibilité iléale de l'azote.

Analysis of variance on the ileal digestibility of nitrogen.

\begin{tabular}{c|c|c|c|c}
\hline Source de variation & ddl & SCE & Carré moyen & F \\
\hline Régime . . . . . . . . . . . & 2 & 4.752 & 2.376 & 1.94 NS \\
Animal . . . . & 4 & 10.265 & 2.566 & $2.10 \mathrm{NS}$ \\
Interaction régime-animal & 8 & 13.991 & 1.749 & $1.43 \mathrm{NS}$ \\
Résiduelle ........ & 30 & 36.665 & 1.222 & \\
\hline \hline
\end{tabular}

B. Cas de la matière sèche

Ces résultats se trouvent dans le tableau 3. Dans ce cas, l'effet régime est significatif au seuil de $1 \%(\mathrm{~F}(2,30)=5,39)$, alors qu'il n'est pas possible de mettre en évidence d'effet lié aux animaux, ni d'interaction régime-animal. Les résultats de l'analyse de covariance, avec comme covariable le taux de coques introduit dans le régime, sont donnés dans le tableau 4.

\section{TABLEAU 3}

Analyse de variance sur la digestibilité iléale de la manière sèche.

Analysis of variance on the ileal digestibility of dry matter.

\begin{tabular}{l|r|r|c|c}
\hline \multicolumn{1}{c|}{ Source de variation } & ddl & SCE & Carré moyen & F \\
\hline Régime $\ldots \ldots \ldots \ldots \ldots \ldots$ & 2 & 88.034 & 44.017 & $8.11++$ \\
Animal . . . . . . . & 4 & 7.329 & 1.832 & $0.34 \mathrm{NS}$ \\
Interaction régime-animal & 8 & 24.671 & 3.084 & $0.57 \mathrm{NS}$ \\
Résiduelle . . . . . . & 30 & 162.718 & 5.424 & \\
\hline \hline
\end{tabular}

\section{Tableau 4}

Analyse de covariance sur la digestibilité iléale de la matière sèche.

Covariance analysis on the ileal digestibility of dry matter.

\begin{tabular}{c|r|r|r|r}
\hline \hline Source de variation & ddl & \multicolumn{1}{|c|}{ SCE } & Carré moyen & \multicolumn{1}{c}{ F } \\
\hline Animal . . . . . . . . & 4 & 7.329 & 1.832 & $0.38 \mathrm{NS}$ \\
Taux de coques . . . . & 1 & 85.042 & 85.042 & $17.42++$ \\
Résiduelle . . . . . & 39 & 190.401 & 4.882 & \\
\hline \hline
\end{tabular}


Les résultats des tableaux 3 et 4 permettent de tester l'hypothèse de linéarité de la régression par comparaison des résiduelles des deux modèles (tabl. 5).

Comme la valeur de $F(9,30)$ est de 1,36 au seuil de $25 \%$, il est possible de conclure qu'il n'y a pas d'interaction digestive pour la matière sèche entre les coques de soja et le son de blé au niveau de l'iléon du porc.

TABleau 5

Test d'une interaction digestive : cas de la matière sèche.

Digestive interaction test : (dry matter).

\begin{tabular}{l|c|c|c|c}
\hline \hline & ddl & SCE & Carré moyen & F \\
\cline { 2 - 5 } & & & & \\
Résiduelle de l'analyse de & & & & \\
covariance ......... & 39 & 190.40 & 4.882 & \\
Résiduelle de l'analyse de & & & & \\
variance ... . . . . & 30 & 162.72 & 5.424 & \\
Réduction de la SCE ... & 9 & 27.68 & 3.076 & $0.57 \mathrm{NS}$ \\
\hline
\end{tabular}

\section{Cas de la matière organique}

Contrairement à la matière sèche et à l'azote, les échantillons des 3 jours ont été poolés pour chaque animal, à l'intérieur de chacun des régimes. Il n'est donc pas possible de tester une éventuelle interaction régime-animal. Les résultats de l'analyse de variance sont récapitulés dans le tableau 6.

Tableau 6

Analyse de variance sur la digestibilité iléale de la matière organique. Analysis of variance on the ileal digestibility of organic matter.

\begin{tabular}{c|c|c|c|c}
\hline \hline Source de variation & ddl & SCE & Carré moyen & F \\
\hline Régime $\ldots \ldots \ldots \ldots \ldots$ & 2 & 17.892 & 8.946 & $9.17+$ \\
Animal . . . . . . . . & 4 & 2.108 & 0.527 & $0.54 \mathrm{NS}$ \\
Résiduelle . . . . . & 8 & 7.805 & 0.976 & \\
\hline \hline
\end{tabular}

TABleau 7

Test de l'hypothèse de linéarité de la régression sur la digestibilité iléale de la matière organique. Testing the linearity of the regression on the ileal digestibility of organic matter.

\begin{tabular}{l|c|c|c|c}
\hline \hline & ddl & SCE & Carré moyen & F \\
\cline { 2 - 5 } Résiduelle de l'analyse de & & & & \\
covariance ........ & 9 & 10.930 & 1.214 & \\
Résiduelle de l'analyse de & 8 & 7.808 & 0.976 & \\
variance ......... & 1 & 3.122 & 3.122 & $3.20 \mathrm{NS}$ \\
Réduction de la SCE ... &
\end{tabular}


A partir du carré moyen résiduel de l'analyse de variance-covariance $(1,21$ avec 9 ddl), il est possible de construire le tableau qui permet de tester l'hypothèse de linéarité de la régression (tabl. 7).

La comparaison de la valeur obtenue $(3,20)$ avec la valeur de $F(1,8)$ au seuil de $10 \%(3,46)$ indique qu'il est possible de rejeter l'hypothèse de non-linéarité à ce seuil.

\section{Cas de la méthionine}

Comme pour la matière organique, les échantillons ont été regroupés par animal et par régime. Les résultats de l'analyse de variance concernant la méthionine sont récapitulés dans le tableau 8.

Sachant que la résiduelle de l'analyse de covariance a un carré moyen de 1,38 avec $9 \mathrm{ddl}$, il est possible de construire le tableau qui permet de tester l'hypothèse de linéarité de la régression (tabl. 9). Cette hypothèse est rejetée dans le cas présent, ce qui signifie qu'il y a existence d'interaction digestive entre le son de blé et les coques de soja, au niveau de la digestibilité iléale de la méthionine chez le porc.

\section{TABleau 8}

Analyse de variance sur la digestibilité iléale de la méthionine.

Analysis of variance on the ileal digestibility of methionine.

\begin{tabular}{c|c|c|c|c}
\hline \hline Source de variation & ddl & SCE & Carré moyen & F \\
\hline Régime $\ldots \ldots \ldots \ldots \ldots \ldots$ & 2 & 19.181 & 9.59 & $25.2++$ \\
Animal . . . . . . . . . & 4 & 1.399 & 0.35 & $0.9 \mathrm{NS}$ \\
Résiduelle . . . & 8 & 3.040 & 0.38 & \\
\hline \hline
\end{tabular}

\section{TABleau 9}

Test d'une interaction digestive: cas de la méthionine.

Digestive interaction test : methionine.

\begin{tabular}{l|c|c|c|c}
\hline \hline & ddl & SCE & Carré moyen & F \\
\cline { 2 - 5 } & & & & \\
Résiduelle de l'analyse de & 9 & 12.43 & 1.38 & \\
covariance . . . . . & & & & \\
Résiduelle de lanalyse de & 8 & 3.05 & 0.38 & $24.7++$ \\
variance . . . . . . & 1 & 9.38 & 9.38 & $24.7+\ldots$ \\
\hline
\end{tabular}

\section{Discussion et conclusion}

La méthode de travail proposée dans cet article permet de tester statistiquement une éventuelle interaction digestive entre deux matières premières distribuées seules ou en association chez un monogastrique. Elle est en partie dérivée de la méthode des 
régressions qui est la méthode la plus fiable pour résoudre une question analogue chez le ruminant (Giger \& Sauvant, 1983), mais qui n'est applicable que si le nombre de régimes est supérieur à 3 dans le cas de 2 constituants et quelle que soit la forme de l'interaction. La méthode proposée, qui est basée sur l'étude des variances résiduelles issues de deux modèles de variance-covariance, peut s'appliquer tout aussi bien aux dispositifs avec ou sans répétition, et même à ceux comportant des effectifs déséquilibrés entre les lots.

Cette méthode présente l'intérêt de faire jouer un rôle analogue aux deux constituants du régime, ce qui n'aurait pas été le cas si la digestibilité mesurée d'un des constituants avait été comparée à celle obtenue par la méthode des différences à partir des mesures effectuées sur les deux autres régimes. De plus, cette méthode est relativement facile à mettre en ouvre et peut même être utilisée avec des machines à calculer portatives, lorsque le nombre de répétitions et d'animaux n'est pas trop élevé et s'il est le même pour chaque animal. Elle permet également de tester les effets liés aux animaux et peut être utilisée avec un plus grand nombre de taux d'incorporation dans le mélange. Dans ce cas, le test de la linéarité de la régression se fera avec un carré moyen de la réduction de la SCE qui aura un nombre de ddl supérieur à 1 (nombre de taux - 2).

Cette méthode, conçue pour répondre au cas type du monogastrique qui reçoit une ration homogène, peut être appliquée dans certains cas aux ruminants, comme celui où ils ingèrent des rations complètes compactées, ce qui leur interdit d'intervenir, par leur tri, sur les taux de fourrage et de concentré ingérés.

\section{Remarque}

Ce travail a été entrepris à l'occasion de l'A.T.P. (action thématique programmée) INRA sur les parois végétales et qui impliquait notamment les laboratoires INRA suivants : Station de Nutrition et Alimentation de l'I.N.A.-P.G. (Paris) ; Laboratoire de Physiologie de la Nutrition du C.R.J. (Jouy-en-Josas).

Reçu en juillet 1987.

Accepté en décembre 1987.

\section{Summary \\ A method for testing digestive interactions between diet components in monogastric animals}

Estimation of the nutritive value of a compound feed is generally based on the additivity of each of its ingredients. However, this approach can only be used in the absence of any significant digestive interaction.

The described method was used to test statistically the possible existence of such an interaction between two ingredients even when using more than two compound feeds and whatever the form of interaction. The method involved two steps : The first one consisted of testing « diet effect " by an analysis of variance which also included an « animal effect ». In the case of repeated 
measurements, it was also possible to test the " animal-diet " interaction. When there was no diet effect, there was no digestive interaction (table 2).

If the "diet effect " was significant, it was necessary to use a second step, i.e. a regression method. The accuracy provided with the analysis of variance over the regression allowed to test the possible existence of a digestive interaction (tables 4 and 5).

This method could also be used when the measurements were not repeated (tables 6 to 9) or when the experimental device was unbalanced. Effects linked to animals or animal-diet interaction could also be easily tested by means of this method.

Key words: Digestive interaction, nutritive value, diet components, pig, monogastric animal.

\section{Références bibliographiques}

Giger S., Sauvant D., 1983. - Comparaison de différentes méthodes d'évaluation du coefficient d'utilisation digestive des aliments concentrés par le ruminant. Ann. Zootech., 32, 215-246.

Laplace J.P., Darcy-Vrillon B., Perez J.M., Henry Y., Giger S., Sauvant D., 1988. Associative effects between two fibre sources on ileal and faecal digestibility of amino acids, energy and cell wall components in growing pigs. Br. J. Nutr., soumis pour publication.

SChNeIDER B.H., Flatt W.P., 1975. - The evaluation of feeds through digestibility experiments, The University of Georgia Press, Athens, (U.S.A.), 423 p.

SHEFFE H., 1959. - The analysis of variance. Wiley, New-York, (U.S.A.), 477 p.

Snedecor G.W., Cochran W.G., 1984. - Méthodes statistiques. Sixième édition. Acta, Paris, (France), $649 \mathrm{p}$. 